\title{
Shape-Conformable, Eco-Friendly Cellulose Aerogels as High-Performance Battery Separators
}

Leila Raafat, ${ }^{a}$ Bernd Wicklein, ${ }^{b}$ Günter Majer, ${ }^{c}$ Timotheus Jahnke, ${ }^{d}$ Achim M. Diem, ${ }^{a}$ Joachim Bill a and Zaklina Burghard ${ }^{\mathrm{a}, *}$

a Institute for Materials Science, University of Stuttgart, Heisenbergstraße 3, 70569 Stuttgart, Germany. E-mail: zaklina.burghard@imw.uni-stuttgart.de

b Instituto de Ciencia de Materiales de Madrid (ICMM), CSIC, Sor Juana Inés de la Cruz 3, 28049 Cantoblanco, Madrid, Spain.

c Max Planck Institute for Intelligent Systems, Heisenbergstraße 3, 70569 Stuttgart, Germany.

d Max Planck Institute for Medical Research, Jahnstraße 29, 61920 Heidelberg, Germany.

\section{Morphology Evaluation}
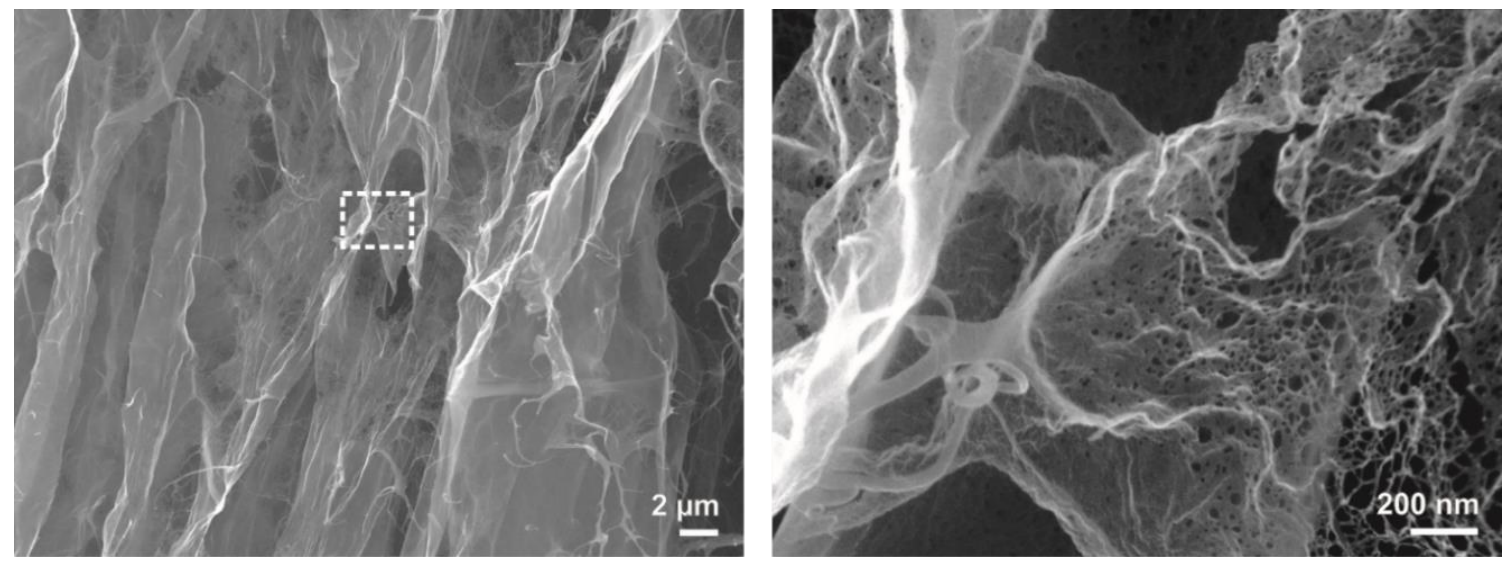

Figure S1. SEM micrographs showing the meso- and macroporosity of the cell-walls.

The porosity of the samples was determined using

$P_{\mathrm{s}}=\left(1-\frac{\rho_{\mathrm{s}}}{\rho_{\text {cel }}}\right) \times 100 \%$,

(Equation S1)

where the density of cellulose $\rho_{\text {cel }}$ is $1460 \mathrm{~kg} \mathrm{~m}^{-3},{ }^{[1]}$ and the apparent density of the sample $\rho_{\mathrm{s}}$ is the ratio of sample weight to its volume.

From the cumulative pore volume $V_{p}$, determined by BET measurements, and the specific sample volume $V_{s}$, the percentage of mesopores $P_{\mathrm{mp}}$ is assessed as described by

$P_{\mathrm{mp}}=\frac{V_{\mathrm{p}}}{V_{\mathrm{s}}} \times 100 \%$

(Equation S2)

The difference between $P_{\mathrm{s}}$ and $P_{\mathrm{mp}}$ can be ascribed to the porosity realized by the channels $P_{\mathrm{c}}$, the macroporosity. 

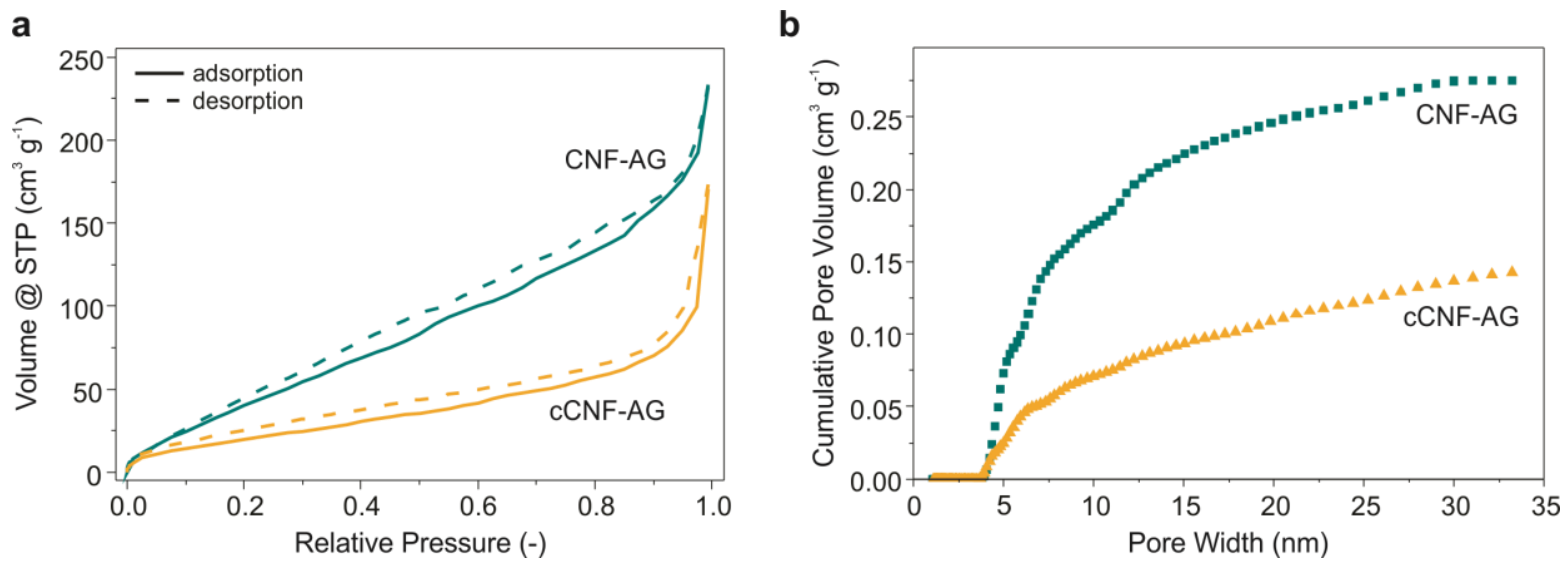

Figure S2. (a) Isotherm obtained by $\mathrm{N}_{2}$ adsorption and desorption of the CNF-AG and cCNF-AG. (b) Cumulative pore volume of the CNF-AG and CCNF-AG.
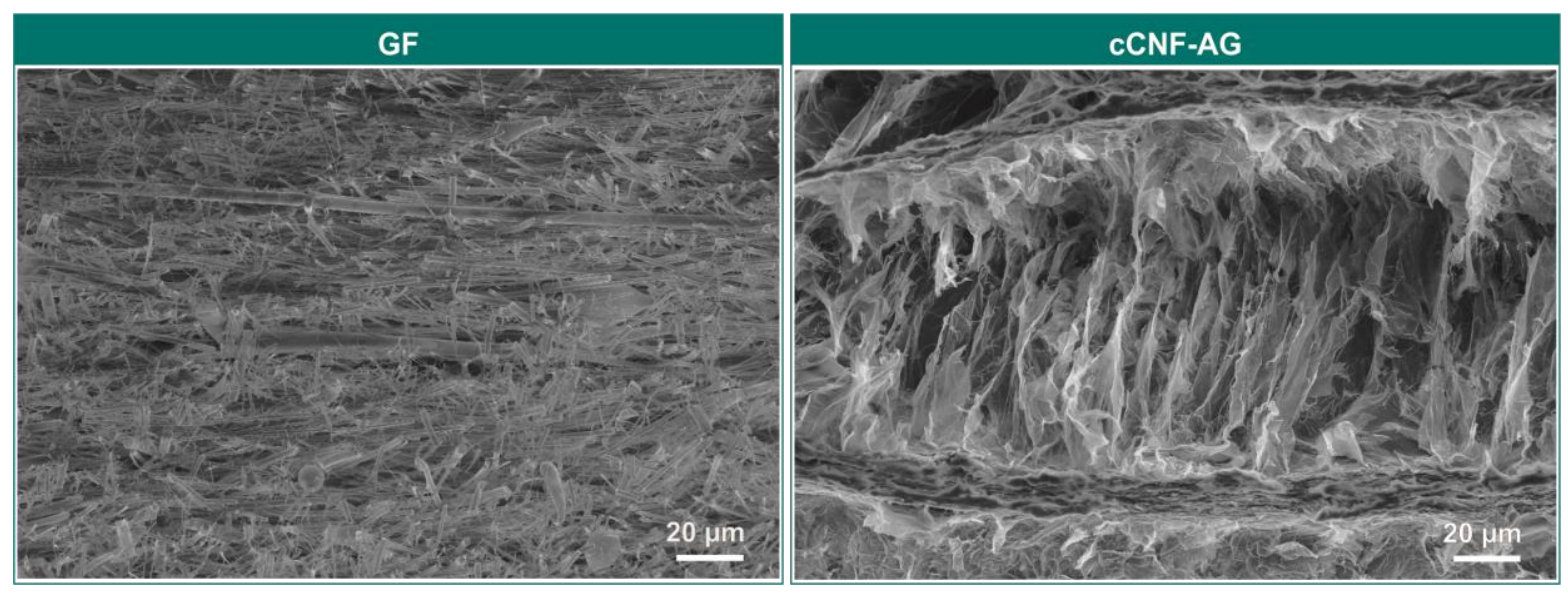

Figure S3. A high magnification of the longitudinal section of the GF and the compressed CNF-AG, showing the densified regions between the porous channel-like domains.

Table S1. $\mathrm{N}_{2}$-sorption results for the CNF-AG and CCNF-AG, where the pore width distribution peaks are indicated by (I), (II) and (III), from highest to lowest, respectively. (II-S) denotes the observed shoulder near peak (II) for the CNF-AG, which is transformed into a peak after compression.

\begin{tabular}{lcclr}
\hline Sample & Cumulative pore volume $\left(\mathrm{cm}^{3} \mathrm{~g}^{-1}\right)$ & BET surface area $\left(\mathrm{m}^{2} \mathrm{~g}^{-1}\right)$ & \multicolumn{2}{l}{ mesopores width (nm) } \\
\hline CNF-AG & 0.276 & 218.740 & (I) & $4.55 \pm 0.10$ \\
& & & (II) & $6.55 \pm 0.10$ \\
& & & (II-S) & $8.35 \pm 0.10$ \\
cCNF-AG & 0.143 & & (III) & $11.55 \pm 0.10$ \\
& & & (I) & $4.55 \pm 0.10$ \\
& & & (II) & $6.55 \pm 0.10$ \\
& & & (III) & $8.35 \pm 0.10$ \\
& & & (IV) & $11.55 \pm 0.10$ \\
\hline
\end{tabular}


Table S2. The apparent density of the CNF-AG and CCNF-AG, as well as the total porosity of the samples and the percentage of the channels and cell-wall porosity.

\begin{tabular}{lllll}
\hline Sample & $\rho_{\mathrm{s}}\left(\mathrm{kg} \mathrm{m}^{-3}\right)$ & $P_{\mathrm{s}}(\%)$ & $P_{\mathrm{c}}(\%)$ & $P_{\mathrm{mp}}(\%)$ \\
\hline CNF-AG & $7.1 \pm 1.3$ & $99.5 \pm 0.1$ & $99.3 \pm 0.1$ & $0.20 \pm 0.04$ \\
cCNF-AG & $71.2 \pm 15.7$ & $95.1 \pm 1.7$ & $94.1 \pm 1.3$ & $1.02 \pm 0.22$ \\
\hline
\end{tabular}

\section{Thermal Analysis}

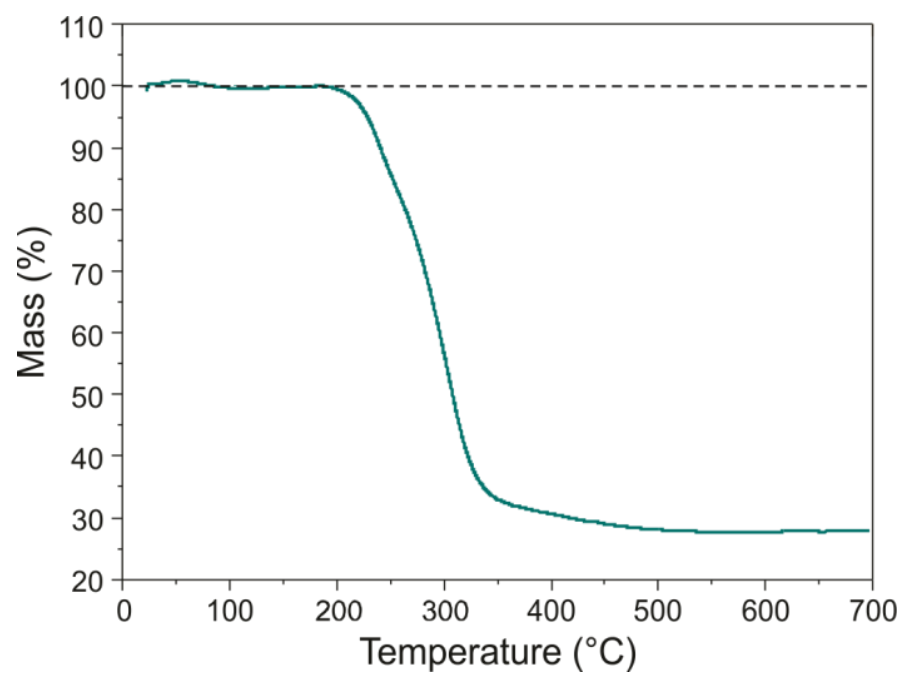

Figure S4. TGA measurement of the CNF-AG.

\section{Saturation Analysis}

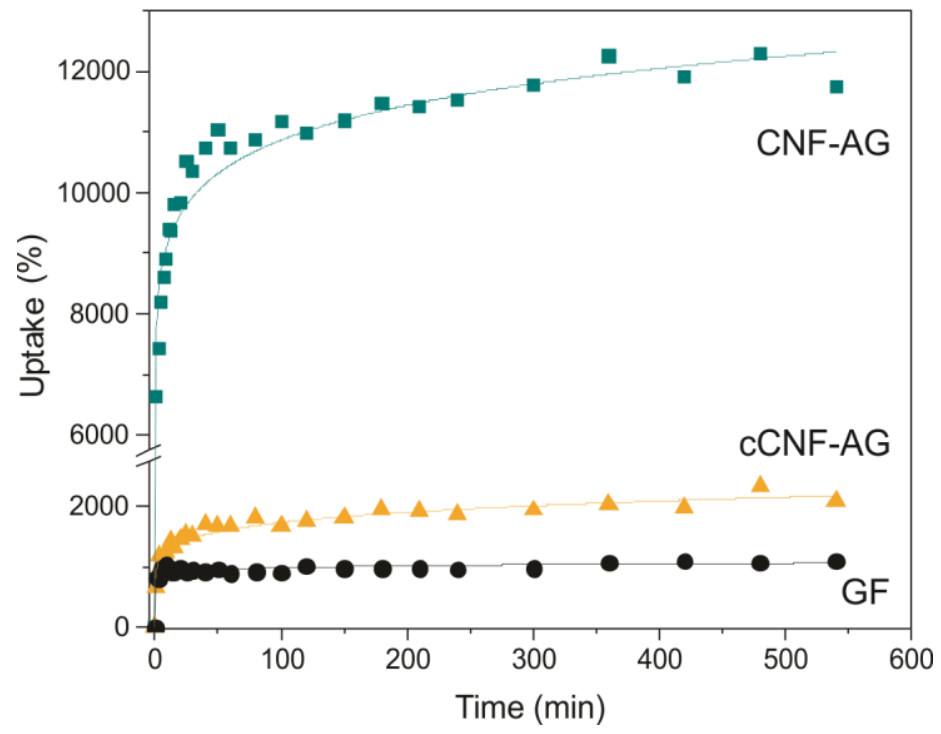

Figure S5. Electrolyte uptake over nine hours for the CNF-AG, CCNF-AG and GF. 
The Chapman-Richards model is described by

$y=a \times\left(1-e^{-b t}\right)^{c}$,

(Equation S3)

where $a$ represents the upper limit (100\%), $b$ the growth rate and $c$ determines the location of the inflection point.

Table S3. Parameters determined for the Chapman-Richards model, $y=a\left(1-e^{-b t}\right)^{c}$, describing the saturation of the different samples using $\mathrm{a}=100 \%$ as it represents the upper limit.

\begin{tabular}{lll}
\hline Sample & $b$ & $c$ \\
\hline CNF-AG & $7.81 \times 10^{-3}$ & $9.75 \times 10^{-2}$ \\
cCNF-AG & $8.20 \times 10^{-3}$ & $1.68 \times 10^{-1}$ \\
GF & $2.86 \times 10^{-4}$ & $3.24 \times 10^{-2}$ \\
\hline
\end{tabular}

Table S4. The required time to reach a certain saturation state for each sample, determined by the obtained Chapman-Richards model.

\begin{tabular}{llll}
\hline Saturation (\%) & \multicolumn{1}{c}{ Time (min) } \\
& CNF-AG & cCNF-AG & GF \\
\hline 10 & $7.1 \times 10^{-9}$ & $1.3 \times 10^{-4}$ & 0 \\
20 & $8.7 \times 10^{-6}$ & $8.2 \times 10^{-3}$ & $3.9 \times 10^{-13}$ \\
30 & $5.6 \times 10^{-4}$ & $9.2 \times 10^{-2}$ & $1.8 \times 10^{-9}$ \\
40 & $1.1 \times 10^{-2}$ & $5.2 \times 10^{-1}$ & $1.8 \times 10^{-6}$ \\
50 & $1.0 \times 10^{-1}$ & 2.0 & $5.0 \times 10^{-4}$ \\
60 & $6.8 \times 10^{-1}$ & 5.9 & $5.8 \times 10^{-2}$ \\
70 & 3.3 & 15.5 & 3.6 \\
80 & 13.7 & 37.4 & 137.9 \\
90 & 53.1 & 92.9 & 12200.7 \\
99.9 & 587.0 & 624.9 & \\
\hline
\end{tabular}




\section{Mechanical Properties}

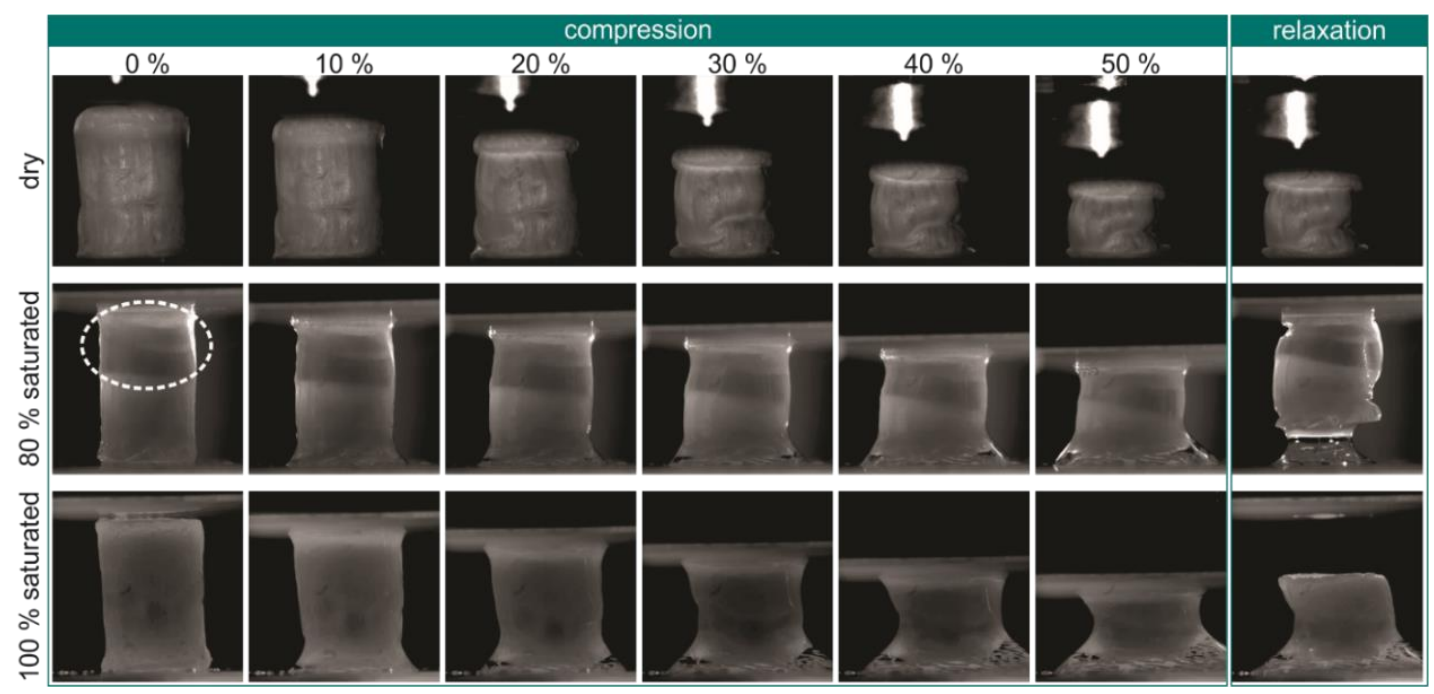

Figure S6. CNF-AG at different compressive strain stages for 0,80 and $100 \%$ saturation. The dry core of the aerogel at $80 \%$ saturation is indicated by a dashed line at $0 \%$ strain.

Table S5. Mechanical Properties of the CNF-AG at different saturation levels.

\begin{tabular}{lllll}
\hline Mechanical Property & Saturation Level & & \\
& $0 \%$ & $60 \%$ & $80 \%$ & $100 \%$ \\
\hline E-Modulus (kPa) & $173.6 \pm 53.5$ & $191.9 \pm 57.4$ & $232.3 \pm 54.0$ & $235.6 \pm 109.7$ \\
Compressive Strength (kPa) & $9.6 \pm 1.2$ & $10.2 \pm 1.8$ & $10.3 \pm 2.5$ & $12.3 \pm 1.4$ \\
Absorbed Energy $\left(\mathrm{kJ} \mathrm{m}^{-3}\right)$ & $440.2 \pm 83.8$ & $569.6 \pm 86.0$ & $585.0 \pm 98.2$ & $596.8 \pm 90.1$ \\
Recovery (\%) & $6.6 \pm 0.6$ & $9.4 \pm 1.1$ & $11.2 \pm 2.9$ & $15.3 \pm 2.5$ \\
\hline
\end{tabular}

Video S1. The static compression and relaxation of the dry aerogel.

Video S2. The static compression and relaxation of the $80 \%$ saturated aerogel.

Video S3. The static compression and relaxation of the $100 \%$ saturated aerogel. 


\section{Electrochemical Investigation}

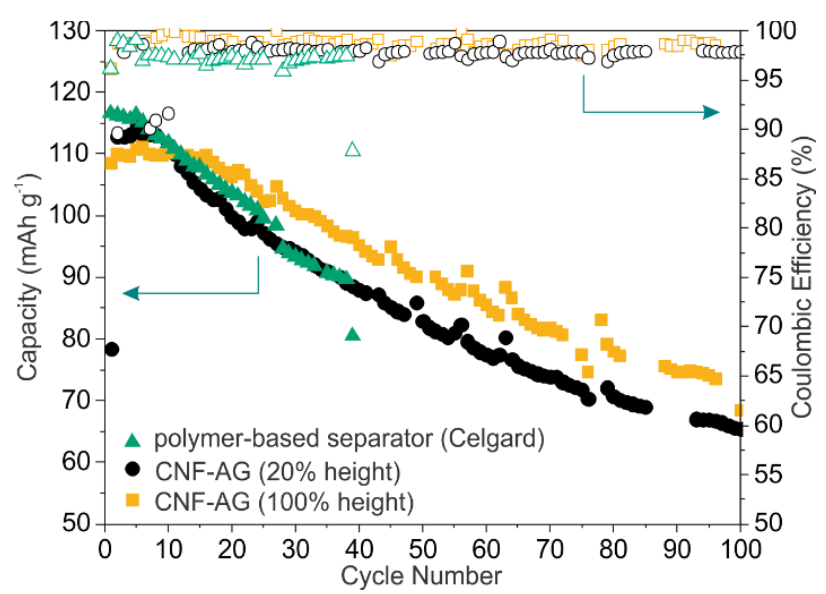

Figure S7. The electrochemical performance of the half-cell Li|separator|LFP at $50 \mathrm{~mA} \mathrm{~g}^{-1}$, using a polymer-based separator, the CNF-AG at full height and another with $20 \%$ of the height $(0.1 \mathrm{~mm})$, showing the achieved capacities and coulombic efficiencies over the cycles.

a

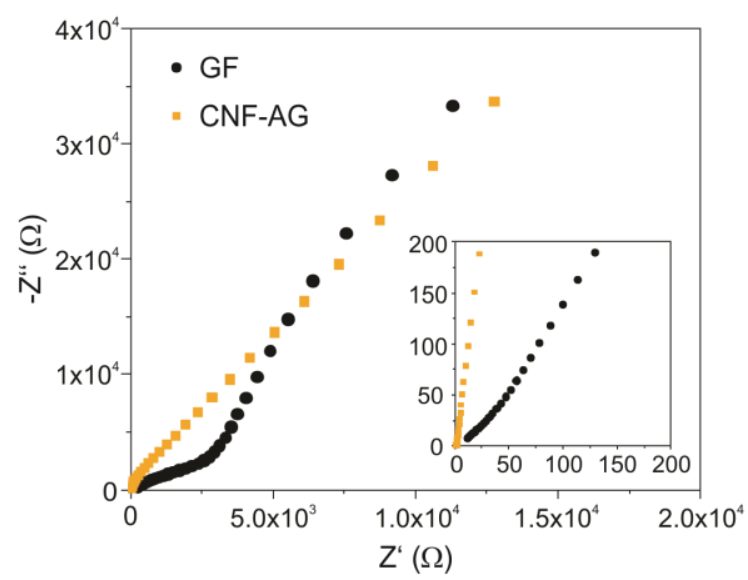

b

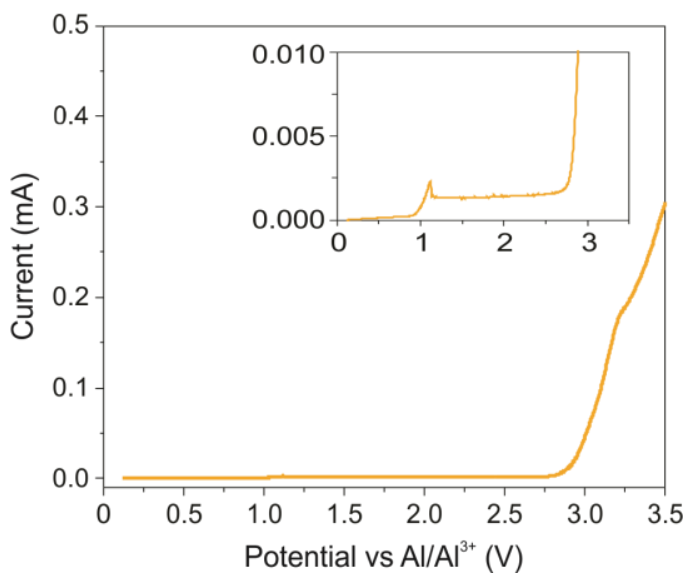

Figure S8. Nyquist plot for the $\mathrm{AlCl}_{3}$ in [EMIM]Cl electrolyte, showing the results for the CNF-AG and GF. (b) The potential window stability of the CNF-AG in conjunction with the electrolyte for AIB. The insets in (a) and (b) are images of high impedance and potential resolution, respectively. 


\section{Ice-Templating}

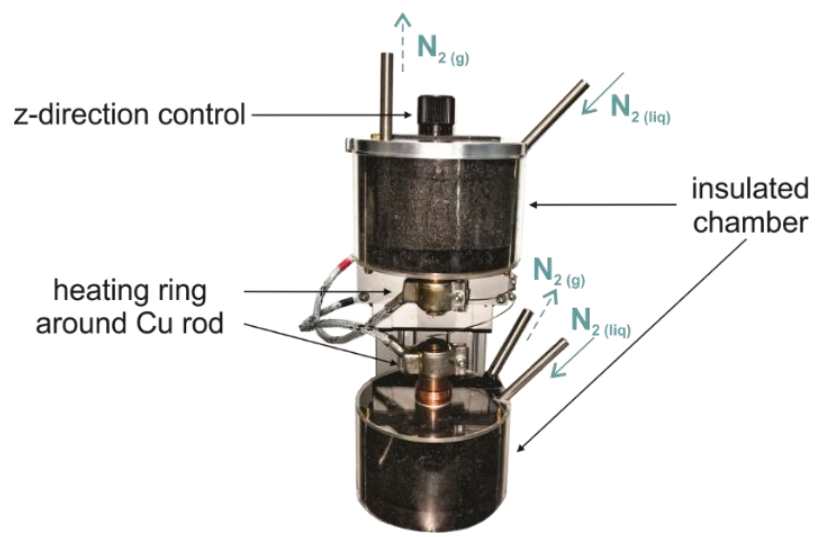

Figure S9. The in-house designed ice-templating device, showing the insulated chambers filled with the liquid nitrogen. The temperature of the $\mathrm{Cu}$ rods is regulated via thermocouples connected to the heating ring.

\section{References}

[1] B. Wicklein, Nat. Nanotechnol. 2015, 10, 277. 